\title{
CALIBRATION AND VALIDATION OF A VOLUME-DELAY FUNCTION USING A GENETIC ALGORITHM
}

\author{
José Elievam Bessa Jr. ${ }^{1 *}$, Vinícius de Magalhães ${ }^{2}$ and Gustavo H. G. Santos ${ }^{3}$ \\ ${ }^{1}$ Department of Transportation and Geotechnical Engineering, Federal University of Minas Gerais, Brazil \\ ${ }^{2}$ Transportation and Traffic Company of Belo Horizonte, Brazil \\ ${ }^{3}$ Department of Transportation Engineering, São Carlos School of Engineering, University of São Paulo, Brazil
}

Received 7 June 2021; received in revised form 16 November 2021; accepted 18 December 2021

\begin{abstract}
In microscopic traffic networks, searching and optimization algorithms are commonly used to calibrate behavioral and performance models of simulators, such as genetic algorithms (GAs). GA applications in macroscopic simulations have not been reported. The aim of this study was to develop a GA to calibrate and validate a volume-delay function (Akçelik) to macroscopically simulate a Brazilian city, Belo Horizonte, in AIMSUN software. Traffic data were used to estimate the capacity, free-flow speed, and parameter $\mathrm{J}$ of the Akçelik function for arterial segments. For other segment types (collectors, locals, and expressways), these data were obtained by the GA. The algorithm application provided higher values of the GA's fitness function (that compares simulated and observed traffic volumes) than those obtained with the parameters suggested in the literature.
\end{abstract}

Keywords: Traffic simulation; Volume-delay function; Genetic algorithm

(C) 2021 Journal of Urban and Environmental Engineering (JUEE). All rights reserved.

\footnotetext{
*Correspondence to: José Elievam Bessa Jr., Tel.: +55 31 97305-9297. E-mail: elievamjr@gmail.com
} 


\section{INTRODUCTION}

Traffic simulations have been carried out by public and private companies to solve problems in urban and rural networks. The progress in personal computers has allowed the development of traffic simulation software capable of generating more complex, accessible, and full alternatives. A traffic simulator can replicate complex situations, while numerical and analytical methods cannot solve problems properly in some cases. In a short time, it can also generate different virtual scenarios, demonstrating them visually and without the need to test them, saving time and resources (FHWA, 2004; Antoniou et al., 2014).

Most traffic simulators were initially developed to model microscopic networks. Macroscopic traffic modeling is usually related to fluid dynamics, where vehicles have similar characteristics and choose the route based on a cost function, which may be related, for example, to the capacity of the streets. The mesoscopic simulation consists of a model with an intermediate degree of complexity between micro- and macroscopic simulations and can be explained, for example, by traffic models such as the speed-flow relationship (Barceló, 2010).

Traffic simulations were separately developed according to the type. The current software can also perform hybrid simulations, in which more than one type of simulation can be performed simultaneously. An application of the hybrid simulation, for example, combines mesoscopic and macroscopic simulations (TSS, 2015).

The calibration and validation of the simulators are commonly carried out manually. Usually, applications are restricted to specific contexts and cannot be expanded to other parts of the city without full restart of the process. Therefore, it is necessary to adjust the parameters of the simulator in a logical and automatic manner for both microsimulated and macrosimulated networks (Spiliopoulou et al., 2015; Punzo and Montanino, 2016; Yu and Fan, 2017; Maheshwary et al., 2020).

For macroscopic networks, simulators, by the determination of traffic zones, centroids, and field counts, estimate the OD matrix of trips based on an initial matrix (seed). In another approach, simulators can assign an OD matrix without the need for adjustment, often resulting from socioeconomic surveys. With the obtained OD matrix, the trips are allocated to the links, which have their costs estimated by volume-delay functions (VDFs), which are important for the choice of routes by the drivers. It is common for these functions to be used according to the standard provided by the simulators or with values suggested in the literature, without the calibration of the models by field data (Aghamohammadi and Laval, 2020; Batista et al., 2021).

In microscopic networks, calibration and validation of simulators have generally been performed to adjust behavioral submodels such as car-following, lanechanging, and gap-acceptance. The genetic algorithm (GA) is a search and optimization tool widely used for the calibration of microscopic networks, such as Paramics (Ma et al., 2007; Ma and Abdulhai, 2012; Zhou and Cai, 2014; Shan et al., 2018), VISSIM (Park and Qi, 2005; Stevanovic et al., 2008; Yu and Fan, 2017; Siam et al., 2018; Gallelli et al., 2019), and TWOPAS (Dowling and Skabardonis, 2006; Moreno et al., 2016).

In literature, despite there are few GA applications for calibration and validation of macroscopic traffic flow models (Spiliopoulou et al., 2015; Shirke et al., 2019), have not been reported other ones to adjust VDF parameters. Thus, the aim of this study was to develop a GA to calibrate and validate a VDF, Akçelik (Akçelik, 1991), that was used to model Belo Horizonte City in AIMSUN software macroscopically.

\section{TRAFFIC ASSIGNMENT AT AIMSUN}

AIMSUN was created in 1986 in Barcelona, Spain. The software was a tool of a research program at the Polytechnic University of Catalonia (UPC). Initially, it was intended to address only microscopic simulations. Since then, several functionalities, such as meso- and macroscopic models, have been included in the software (Aimsun, 2021).

In AIMSUN, to statically carry out travel assignments of an OD matrix, it is necessary to determine a cost function for the links in the segment network. In the simulator, one of three types of cost function can be used: i) VDF, ii) turn penalty function, and iii) junction delay function (TSS, 2015). There are five different types of methods to perform a static traffic assignment: i) all-or-nothing, ii) incremental, (iii) Method of Successive Averages (MSA), iv) FrankWolfe, and v) stochastic methods.

In this study, the Frank-Wolfe traffic assignment method was used. The method is based on the concept of user balance, which assumes that travelers try to minimize the individual travel time by choosing the route that appears to be shortest. The method is formulated from the principle of Wardrop, which assumes that the travel time of all routes is equal to or smaller than the time of any other route for an OD pair (Barceló, 2010). The VDF type was chosen for the cost function but modified in relation to that used as default by AIMSUN, as discussed in the following section. 


\section{Volume-delay functions (VDFs)}

The VDFs are mathematical relationships used in the traffic assignment step of the four-step demand forecasting model. These functions represent the delay factor related to that obtained under free-flow conditions (15). The VDF represents the total travel time in each segment. It is related to the segment capacity to the traffic volume transiting within it in each time period, both expressed in vehicles per hour:

$$
t(v)=t_{0} \cdot f\left(\frac{v}{c}\right)
$$

where $t_{0}$ is the travel time in free flow $(\mathrm{h} / \mathrm{km}), v$ is the traffic flow (veh/h), and $c$ is the capacity $(\mathrm{veh} / \mathrm{h})$.

The relationship between these values in the function always appears by the ratio $v / c$. The VDFs use a factor of increase in the travel time that an individual would have to cross a segment. It increases exponentially under congestion conditions when the traffic demand is larger than the segment capacity when $(v / c)>1$. This is valid because, in the real world, a longer time period is required to travel a route within the city during peak hours when there are too many vehicles on the streets.

Different types of VDFs have been used. The oldest and most used is the VDF of the Bureau of Public Segments (BPR) (BPR, 2015), which has a parabolic form:

$$
f\left(\frac{v}{c}\right)=\left[1+\alpha \cdot\left(\frac{v}{c}\right)^{\beta}\right],
$$

where $\alpha$ and $\beta$ are calibration constants based on the characteristics of the path. The values of the BPR function tend to infinity according to a vertical asymptote for supersaturation values, when $(v / c)>1$. In addition, the function can still have virtually zero sensitivity at low traffic levels.

The VDF of Akçelik (Akçelik, 1991) tends to approximate the results predicted analytically more closely through the queuing theory, particularly in situations of congestion. The VDF of Akçelik is

$$
t_{A k s}=t_{0} \cdot\left\{1+0,25 \cdot \frac{T}{t_{0}} \cdot\left[\frac{v}{c}-1+\sqrt{\left(\frac{v}{c}-1\right)^{2}+\frac{8 \cdot J}{c \cdot T} \cdot \frac{v}{c}}\right]\right\},
$$

where $t_{A k k_{\xi}}$ is the total travel time $(\mathrm{h} / \mathrm{km}), T$ is the duration of the analysis interval (h), and $J$ is the calibration coefficient.

According to Akçelik (1991), the parameter $J$ is expressed by the product of two other parameters, $k$, which depends on the randomness of the process of arrivals and attendance on a route where queues are formed, and $p$, the number of elements that can cause delays in a segment, as the number of intersections per unit distance. An appropriate value for $k$ in a network with isolated signals is 0.6 , while $k$ is equal to 0.3 at locations with coordinated signals.

As a positive aspect of the Akçelik VDF, the use of only one calibration parameter $(J)$ should be emphasized if the capacity and free-flow speed (FFS) are obtained in the field. Therefore, the Akçelik curve seems to be more adequate than the BPR function in integrating the traffic assignment method, which justifies the choice in this study. In AIMSUN, the BPR function is standard, but the version of the simulator used in this study, version 8.1.3, integrated with the application programming interface, allows programming (in Python) of the VDF with the Akçelik model.

\section{TRAFFIC DATA COLLECTION}

To estimate the segment capacity, FFS, and parameter $J$ of some segments and validate the assignment of traffic in the network, traffic data were obtained from 130 detectors installed in the city of Belo Horizonte. Each detector can identify three types of vehicles: light (automobiles), motorcycles, and heavy vehicles (trucks or buses). Each detector can determine the exact time and speed at which each vehicle is registered. The data of all vehicles that passed through these detectors were considered during the Tuesdays, Wednesdays, and Thursdays in August 2015, considered typical days, with peaks in the morning and end of the afternoon. Fig. 1 shows the locations of the detectors scattered throughout the city.

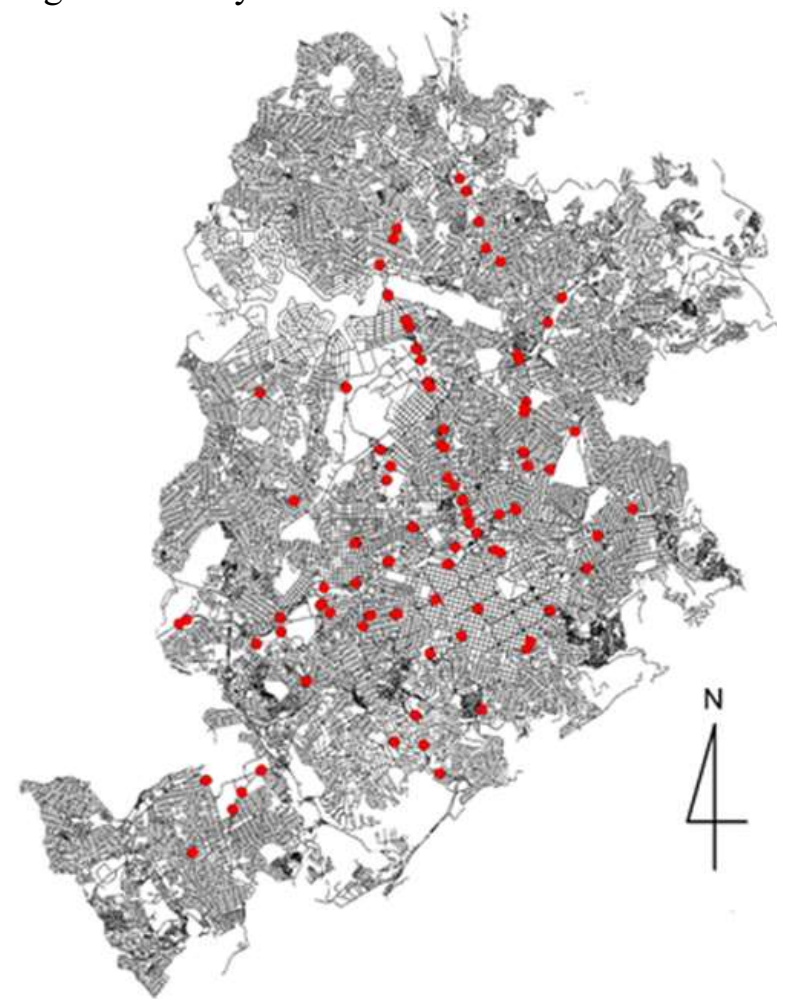

Fig. 1 Locations of traffic detectors in Belo Horizonte. 
Data from each of the 130 detectors were aggregated at 15 -min intervals. An equivalent volume of $0.33 \mathrm{PCU}$ (passenger car unit) was used for motorcycles, while 2.5 PCU for heavy vehicles. Thus, the equivalent traffic flow for each detector was calculated. Considering the traffic data of the detectors, the software SPD_CAL, developed by Van Aerde and Rakha (1995), was used to obtain the Van Aerde flow speed density model. This software uses an iterative heuristic method to calibrate the curves by four parameters, FFS, speed in capacity, capacity, and jam density.

According to Rakha and Crowther (2002), the Van Aerde traffic model is a combination of two simplified models. The simplest of them, the Greenshields model, requires two calibration parameters, the FFS and capacity or jam density. The second model, the pipe carfollowing model, requires three calibration parameters, FFS, jam density, and driver sensitivity factor. The Van Aerde model has a higher level of freedom to reflect different traffic behaviors among several characteristics of the system.

Among the 130 traffic detectors, only 20 had an adequate dispersion of points to obtain the Van Aerde model reaching capacity. These detectors were positioned mainly in the avenues Amazonas, Antônio Carlos, and Cristiano Machado, considered as arterial types. In many cases, the data had a similar dispersion.

Akçelik (1991) suggested $J$ values according to the type of segment, as shown in Table 1. The report does not present a formulation to find $J$ based on traffic variables obtained in the field, such as FFS and capacity, for urban segments. However, it is possible to estimate the shape of the Akçelik function using the flow-speed relationship based on the premise that the curves must be similar before reaching the capacity. With the values of FFS and capacity of the Van Aerde model, the form of the Akçelik function is determined by the parameter $J$. An example of such an application is shown in Fig. 2. Two subtypes of functions for arterial pathways were determined:

- Arterial 1: capacity $=1300 \mathrm{cp} / \mathrm{h} / \mathrm{ln}, \mathrm{FFS}=55$ $\mathrm{km} / \mathrm{h}$, and $\mathrm{J}=0.5$; and

- Arterial 2: capacity $=850 \mathrm{cp} / \mathrm{h} / \mathrm{ln}, F F S=50$ $\mathrm{km} / \mathrm{h}$, and $J=1.0$.

Table 1. Default values of calibration parameters

\begin{tabular}{lccc}
\hline Type & Capacity $(\mathrm{cp} / \mathrm{h} / \mathrm{ln})$ & $F F S(\mathrm{~km} / \mathrm{h})$ & $J$ \\
\hline Expressway & 1800 & 100 & 0.20 \\
Collector 1 & 900 & 60 & 0.80 \\
Collector 2 & 900 & 60 & 0.80 \\
Local 1 & 600 & 40 & 1.60 \\
Local 2 & 600 & 40 & 1.60 \\
\hline
\end{tabular}

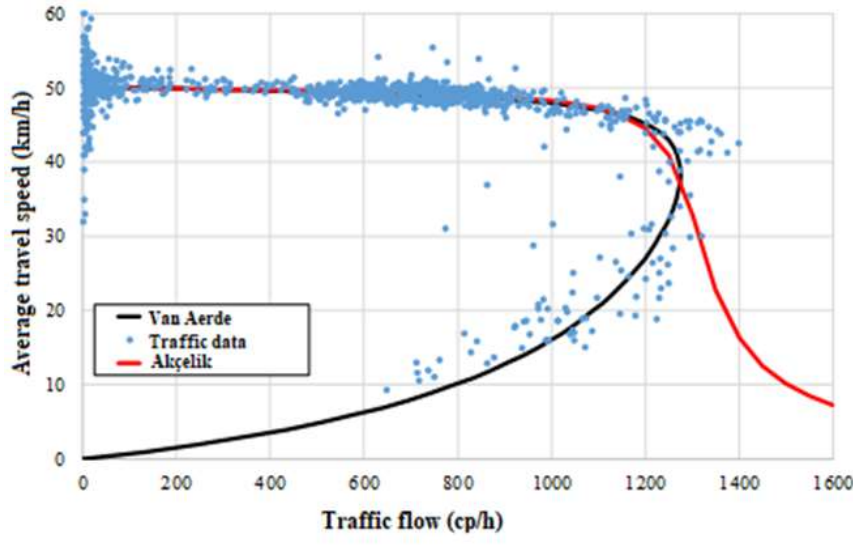

Fig. 2 Van Aerde model obtained from traffic data.

As detectors were not installed on the other types of segments, expressways, collectors, and locations, a GA was applied to find the parameter $J$, capacity, and FFS.

\section{GA APPLICATION AND TRAFFIC ASSIGNMENT}

The simulated network was georeferenced and had several characteristics, such as different segment hierarchies, different numbers of lanes, permitted and forbidden conversions, maximum established speed, and bus lines. The modeled network had 36,201 intersections and extension of $16,695 \mathrm{~km}$. The main avenues of cities in the Metropolitan Region of Belo Horizonte (RMBH) were also added to represent the flow of vehicles between these regions and Belo Horizonte.

It is practically impossible to generate a traffic assignment model in which all travel times between OD pairs are equal. Therefore, to assign traffic statically to AIMSUN, it is important to know the stopping criterion of the assignment algorithm: maximum number of iterations or minimum "relative gap," which represents the mean difference of the travel times between the OD pairs. In this study, 50 iterations were employed or relative gap of at least $3 \%$ as the stopping criterion.

\section{OD Matrix}

The OD matrix is an estimate of the volume and characteristics of the population displacements of a region between the generation and travel attraction areas over a given time interval. The OD matrix of the RMBH was obtained based on a socioeconomic survey. The main objective of the OD survey was to identify the mobility needs of the RMBH population and combine such needs with the socioeconomic characteristics of this population. Approximately $1 \%$ of the residents of households located in the 34 municipalities were interviewed (in total, 39,958 questionnaires; 19,360 of them were in Belo Horizonte). The obtained OD matrix 
was integrally modeled using AIMSUN without adjustments.

\section{GA Application}

To calibrate and validate the Akçelik model, a GA was used with similar characteristics to other applications in a microscale network to calibrate the parameters of behavioral models (Moreno et al., 2016) and performance models (Bessa Jr. and Setti, 2018).

The method consists of randomly producing an initial population of individuals (solutions) that evolve over generations based on their degree of adaptation to the environment (fitness function). For the population to evolve, it is necessary that the best individuals (better adapted) transmit their genetic material to their children (through crossover). In addition, other genetic operators are used, such as mutation of their genes (part of an individual) and predation (or predatory elimination) of those individuals less adapted to the environment, leading to others produced at random. Further details on the use of GAs to calibrate traffic simulators can be obtained from Bessa Jr. e Setti (2018).

In this study, the developed GA sought a solution (individual) whose values of $J$, FFS, and capacity of the VDFs produced simulated traffic volumes close to those obtained by the 130 detectors studied for a certain time period. Because GA is a technique that requires simulation of the same network many times (with different solutions), the segment network used in the calibration was reduced to simulate faster (Fig. 3). The search range of the parameters is presented in Table 2, established based on the parameters suggested by Akçelik (1991), as shown in Table 1.

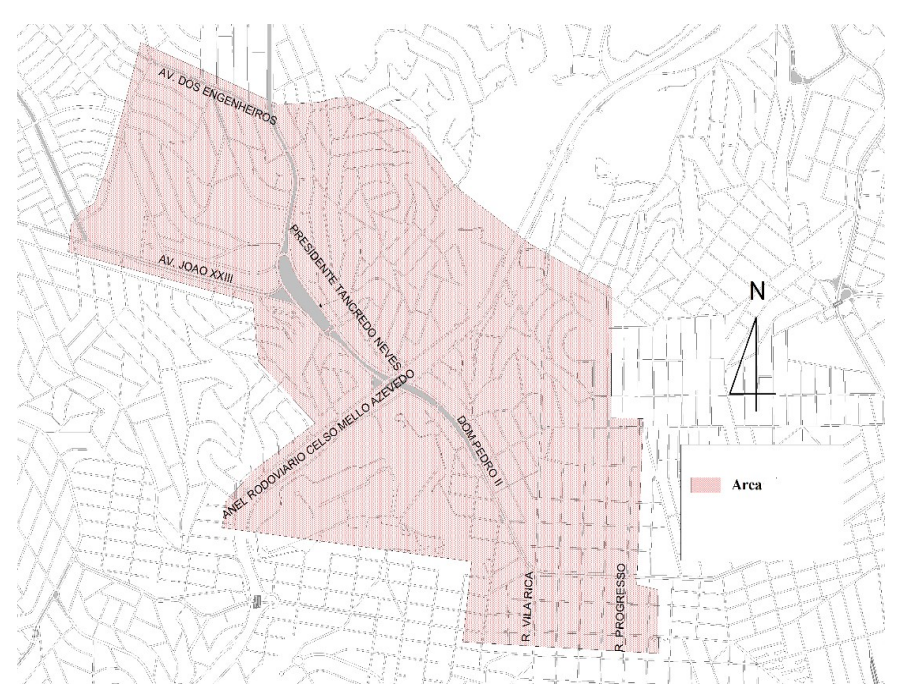

Fig. 3 Network used in the GA application.
Table 2. Range of the GA calibration parameters

\begin{tabular}{lllllll}
\hline & \multicolumn{2}{l}{$\begin{array}{l}\text { Capacity } \\
(\mathrm{cp} / \mathrm{h} / \mathrm{ln})\end{array}$} & $\begin{array}{l}F F S \\
(\mathrm{~km} / \mathrm{h})\end{array}$ & & $J$ & \\
\cline { 2 - 7 } Type & Min & Max & Min & Max & Min & Max \\
\hline Expressway & 1000 & 2000 & 60 & 120 & 0.10 & 0.60 \\
Collector 1 & 700 & 1100 & 40 & 70 & 0.60 & 1.20 \\
Collector 2 & 700 & 1100 & 40 & 70 & 0.60 & 1.20 \\
Local 1 & 400 & 800 & 30 & 50 & 1.20 & 2.00 \\
Local 2 & 400 & 800 & 30 & 50 & 1.20 & 2.00 \\
\hline
\end{tabular}

The GA population was composed of 20 individuals. The stopping criterion of the algorithm was the maximum number of generations equal to 50 . A crossover with the selection criterion of elitism type and diversity (predation and mutation) criteria equal to $30 \%$ and $20 \%$, respectively, was applied after every two generations. The fitness function was the root of the mean square error (RMSE) between the observed and simulated traffic volumes:

$$
R M S E=\sqrt{\frac{1}{n} \sum_{i=1}^{n}\left(x_{i}-y_{i}\right)^{2}},
$$

where $n$ is the total number of detectors, $y_{i}$ is the $i^{\text {th }}$ simulated traffic volume, and $x_{i}$ is the $i^{\text {th }}$ traffic volume observed in the field.

The RMSE choice is justified by the larger penalization of the large differences between the observed and simulated volumes (Hollander and Liu, 2008). The network simulation was carried out considering the peak hour in the morning, between 7 am and 8 am. The OD matrix was adjusted for the population displacement data for this period.

Using the default parameters of the VDF (Table 1), an RMSE value of 462 was obtained for the assignment subnetwork. This value is considered good (low) and was obtained by the simulator because it is a relatively small segment network of $111 \mathrm{~km}$ and 301 intersections. The choice for this network is justified because it contains all types of routes to be calibrated, with field data sufficient for the traffic assignment.

The application of the best solution obtained by the GA (Table 3) in the traffic assignment with AIMSUN provided an RMSE value of 366, 21\% lower than that obtained with the default values of the parameters. Therefore, although the use of the default parameters provided a low value for the fitness function, the GA application improved the traffic assignment, providing traffic volume assignments closer to the observed traffic volumes.

Regarding the results of the calibrated parameters, the FFS, capacity, and $J$ were consistent. When the first two values were larger, the third was smaller. We also note the values for the collector and local roads, with 
Table 3. Best solution obtained by the GA

\begin{tabular}{lccc}
\hline Type & Capacity $(\mathrm{cp} / \mathrm{h} / \mathrm{ln})$ & $F F S(\mathrm{~km} / \mathrm{h})$ & $J$ \\
\hline Expressway & 1780 & 87 & 0.44 \\
Collector 1 & 1090 & 62 & 0.79 \\
Collector 2 & 800 & 47 & 1.11 \\
Local 1 & 440 & 32 & 1.30 \\
Local 2 & 510 & 38 & 1.70 \\
\hline
\end{tabular}

two subtypes for each of them. Parameters with different values were obtained within each segment hierarchy, which supports the hypothesis that it is necessary to find more than one VDF for each type of route.

Using the default parameters listed in Table $\mathbf{1}$ for the traffic assignment of the entire RMBH in AIMSUN, the obtained RMSE value was 1,390. When the calibrated parameters were used, an RMSE value of 1,279 was obtained, which was $8 \%$ lower than that when default values were used. The RMSE values obtained in the validation step were higher than those in the calibration step, because the traffic network is considerably larger in the validation stage, which is a more complex problem to be solved. Nevertheless, the improvement in fitness of $8 \%$ with calibrated values suggests that the method provides a more efficient traffic assignment than that when parameters suggested in the literature are used.

\section{CONCLUSIONS AND RECOMMENDATIONS}

This study aimed to develop a GA to estimate the calibration parameters of the Akçelik VDF for traffic assignment with AIMSUN. This type of GA application was not reported in the literature, although it is common to use GAs to adjust behavioral parameters in microsimulated networks.

The results of the GA application showed an improvement in the RMSE fitness function in both calibration and validation steps in relation to the results obtained with the default parameters. The levels of improvements at each stage were different, as these networks have different sizes and complexities. It is recommended to analyze other fitness functions to continue the analysis of the calibration parameters obtained with the GA application. Hollander and Liu (2008) presented a list of fitness functions candidates for the proposed analysis.

To improve the obtained results, it would be important to obtain more traffic data close to the capacity within the $\mathrm{RMBH}$, if possible, with data that do not originate from electronic surveillance, largely influenced by the user's behavior when viewing the equipment. It is recommended to apply the proposed method with the GA in other areas of Belo Horizonte to perform the calibration of the parameters, as well as in other cities, with sizes of Belo Horizonte and mediumsize cities.

It is of interest to apply the developed GA to calibrate other VDFs and compare the results to those of the GA application to calibrate the Akçelik function. To obtain more reliable results, it is also recommended to assess whether the OD matrix obtained from socioeconomic surveys need not be adjusted and reevaluated. Another method of assigning static traffic, such as the "all-or-nothing" method or incremental method, can be evaluated.

Acknowledgment This work was supported by the Minas Gerais Research Foundation (FAPEMIG grant number APQ-03779-16); the National Council for Scientific and Technological Development (CNPq grant numbers 312651/2018-0 and 439250/2018-8); and the Coordination for the Improvement of Higher Education Personnel (CAPES).

\section{REFERENCES}

Aghamohammadi, R. and J. A. Laval (2020) Dynamic traffic assignment using the macroscopic fundamental diagram: A Review of vehicular and pedestrian flow models. Transportation Research Part B, v. 137, p. 99-118. doi: 10.1016/j.trb.2018.10.017

Aimsun (2021) Aimsun History. Siemens. Available at: $<$ https://www.aimsun.com/about-aimsun/>.

Akçelik, R. (1991) Travel time functions for transport planning purposes: Davidson's function, its time-dependent. Australian Segment Research, v. 21, n. 3, p. 49-59.

Antoniou, C. et al. (2014) Traffic simulation: case for guidelines The Multitude project. Joint Research Centre of the European Commission.

Barceló, J. (2010) Fundamentals of Traffic Simulation. Department of Statistics \& Operations Research Universitat Politècnica de Catalunya, Barcelona.

Batista, S. F. A., L. Leclercq, and M. Menéndez (2021) Dynamic Traffic Assignment for regional networks with traffic-dependent trip lengths and regional paths. Transportation Research Part $C$, v. 127, 103076. doi: 10.1016/j.trc.2021.103076

Bessa Jr., J. E., and J. R. Setti (2018) Evaluating Measures of Effectiveness for Quality of Service Estimation on Two-Lane Rural Highways. Journal of Transportation Engineering, Part A: Systems, v. 144, n. 9, 04018056. doi: 10.1061/JTEPBS.0000178

BPR (1964) Traffic Assignment Manual. U.S. Department of Commerce, Urban Planning division, Washington, D.C., EUA.

Dowling, R., and A. Skabardonis (2006) Urban Arterial Speed-Flow Equations for Travel Demand Models. Innovations in Travel Demand Modeling: Summary of a Conference. Transportation Research Board.

FHWA (2004) Traffic Analysis Toolbox Volume III: Guidelines for Applying Traffic Microsimulation Modeling Software. Publication No. FHWA-HRT-04-040. US Department of Transportation, Federal Highway Administration.

Gallelli, V., G. Guido, A. Vitale, and R. Vaiana (2019) Effects of calibration process on the simulation of rear-end conflicts at roundabouts. Journal of Traffic and Transportation Engineering, v. 6, n. 2, p. 175-184. doi: 10.1016/j.jtte.2018.03.006 
Hollander, Y., and R. Liu (2008) The principles of calibrating traffic microsimulation models. Transportation, Springer Science and Business Media, LLC, v. 35, p. 347-362. doi: 10.1007/s11116$\underline{007-9156-2}$

Ma, T., and B. Abdulhai (2012) Genetic Algorithm-based Optimization Approach and generic tool for calibration traffic microscopic simulation parameters. Transportation Research Record, n. 1800, TRB, National Research Council, Washington, D.C., p. 6-15. doi: 10.3141/1800-02

Ma, J., H. Donge, and H. M. Zhang (2007) Calibration of microsimulation with heuristic optimization methods. Transportation Research Record, n. 1999, TRB, National Research Council, Washington, D.C., p. 208-217. doi: $\underline{10.3141 / 1999-22}$

Maheshwary, P., K. Bhattacharyya, B. Maitra, M. Boltze (2020) A methodology for calibration of traffic micro-simulator for urban heterogeneous traffic operations. Journal of Traffic and Transportation Engineering, v. 7, n. 4, p. 507-519. doi: 10.1016/j.jtte.2018.06.007

Moreno, A. T., C. Llorca, S. S. Washburn, J. E Bessa Júnior, D. K. Hale and A. Garcia (2016) Modification of the Highway Capacity Manual two-lane highway analysis procedure for Spanish conditions. Journal of Advanced Transportation, v. 50, p. 16501665.

Park, B., and H. Qi (2005) Development and evaluation of a procedure for the calibration of simulation models. Transportation Research Record, n. 1934, TRB, National Research Council, Washington, D.C., p. 208-217. doi: $\underline{10.1177 / 0361198105193400122}$

V. Punzo, and M. Montanino (2016) Speed or spacing? Cumulative variables, and convolution of model errors and time in traffic flow models validation and calibration. Transportation Research Part $B$, v. 91, p. 21-33. doi: 10.1016/j.trb.2016.04.012

Rakha H., and B. Crowther (2002) Comparison of Greenshields, Pipes, and Van Aerde Car-following and Traffic Stream Models. Transportation Research Record, n. 1802, p. 248-262. doi: $10.3141 / 1802-28$
Shan, X., P. Hao, K. Boriboonsomsin, G. Wu, M. Barth, and X. Chen (2018) Partially limited access control design for special-use freeway lanes. Transportation Research Part A, v. 118, p. 25-37. doi: 10.1016/j.tra.2018.09.002

Shirke, C., A. Bhaskar, and E. Chung (2019) Macroscopic modelling of arterial traffic: An extension to the cell transmission model. Transportation Research Part C, v. 105, p. 54-80. doi: 10.1016/j.trc.2019.05.033

Siam, M. R. K., S. Nasrin, M. Hadiuzzaman, S. M. Muniruzzaman, and N. Haque (2018) VISCAL: Heuristic algorithm based application tool to calibrate microscopic simulation parameters. Journal of Traffic and Transportation Engineering, v. 5, n. 1, p. 28-43. doi: 10.1016/j.jtte.2017.04.004

Spiliopoulou, I. Papamichail, M. Papageorgiou, I. Tyrinopoulos, and J. Chrysoulakis (2015) Macroscopic traffic flow model calibration using different optimization algorithms. 4th International Symposium of Transport Simulation-ISTS'14, 1-4 June 2014, Corsica, France. doi: 10.1016/j.trpro.2015.03.012

Stevanovic, J., A. Stevanovic, P. T. Martin, and T. Bauer (2008) Stochastic optimization of traffic control and transit priority settings in VISSIM. Transportation Research Part $C$, v. 16, p. 332-349. doi: 10.1016/j.trc.2008.01.002

TSS (2015) AIMSUN User's Manual - Version 8.1. Transport Simulation System - TSS.

Van Aerde M., and H. Rakha (1995) Multivariate Calibration of Single-Regime Speed-Flow-Density Relationships. Proceedings of Vehicle Navigation and Information Conference, IEEE, Piscataway NJ, USA, p. 334-341.

Yu, M., W. D. Fan (2017) Calibration of microscopic traffic simulation models using metaheuristic algorithms. International Journal of Transportation Science and Technology, v. 6, p. 63-77. doi: 10.1016/j.ijtst.2017.05.001

Zhou, Z., and M. Cai (2014) Intersection signal control multiobjective optimization based on genetic algorithm. Journal of Traffic and Transportation Engineering, v. 1, p. 153-158. 